\section{A utilização da Internet na notificação dos defeitos congênitos na Declaração de Nascido Vivo em quatro maternidades públicas do Município de São Paulo, Brasil}

\author{
Use of the Internet to report congenital \\ malformations on birth certificates at four public \\ maternity hospitals in the city of São Paulo, Brazil
}

\author{
1 Departamento de \\ Morfologia e Genética, \\ Universidade Federal de São \\ Paulo, São Paulo, Brasil. \\ Correspondência \\ P. D. R. De Nicola \\ Departamento de Morfologia \\ e Genética, Universidade \\ Federal de São Paulo. \\ Al. Jaú 1592, apto. 11, \\ São Paulo, SP \\ 01420-002, Brasil. \\ pablodenicola@gmail.com
}

\begin{abstract}
The aim of this study was to improve the completion of item 34 on birth certificates at four maternity hospitals in the city of São Paulo, Brazil, in the year 2008. The database of the Municipal Health Department's Information System on Live Births was used to monitor trends in reporting birth defects. An electronic web-based medical record was used to refer indeterminate cases to a leading medical genetics referral center. The electronic medical record contained the patient history, physical examination, and photographs of the newborn. Four maternity hospitals were assessed, with a total of 10,000 births during the year. None of the four hospitals had a staff geneticist. According to the Information System on Live Births, there was an increase in the number of birth defects reported by the four maternity hospitals when compared to previous years and to records for the city of São Paulo as a whole. Based on the findings, the web-based referral and counter-referral method proved efficient.
\end{abstract}

Congenital Abnormalities; Disease Notification; Birth Certificates; Internet; Information Systems
Pablo Domingos Rodrigues De Nicola 1 Mirlene Cecília Soares Pinho Cernach 1 Ana Beatriz Alvarez Perez 1 Decio Brunoni 1

\section{Introdução}

Os defeitos congênitos dos seres humanos atingem cerca de $2 \%$ a $3 \%$ de todos os nascidos vivos. Do ponto de vista biológico, representam um grupo heterogêneo de distúrbios do desenvolvimento embrio-fetal, sendo cerca de $60 \%$ dos casos de causa desconhecida. As de etiologia genética são as mais conhecidas e abrangem as cromossômicas (6\%) e as malformações com herança mendeliana (20\%). As de etiologia dependentes de fatores ambientais ou de interação gene-ambiente são mais numerosas e mais difíceis de serem reconhecidas $1,2,3$.

Em países onde a mortalidade infantil é baixa por causa do controle mais efetivo de causas sócio-ambientais, os defeitos congênitos representam importante contingente na proporção dos óbitos em menores de um ano. A prevenção, desde que conhecidas as causas, faria diminuir mais os coeficientes de mortalidade infantil 4 . Nos países desenvolvidos, os defeitos congênitos são a primeira causa de mortalidade neonatal, gerando um grande impacto nos serviços de saúde. Nos países em desenvolvimento, a principal causa de morbi-mortalidade no período neonatal ainda são as infecções pela falta de assistência pré-natal e perinatal, fazendo que os defeitos congênitos fiquem em segundo plano na atenção aos cuidados de saúde ${ }^{4,5}$.

Com a queda da mortalidade infantil por doenças infecciosas, parasitárias e respiratórias, 
houve um aumento da participação relativa dos defeitos congênitos nas mortes infantis, pois à medida que as outras causas de morte são controladas, eles assumem um papel proporcionalmente maior. Portanto, avanços nos cuidados de saúde e saneamento básico levaram ao declínio das doenças infecciosas e causadas por desnutrição, e, por isso, os defeitos congênitos ganharam grande importância na mortalidade perinatal 6 .

O Ministério da Saúde, considerando a existência de falhas do ponto de vista quantitativo (cobertura) no registro de nascidos vivos, no Brasil, e a impossibilidade de conhecer a distribuição dos nascidos vivos segundo algumas variáveis importantes sob a ótica clínico-epidemiológica (peso ao nascer, índice de Apgar, duração da gestação, tipo de parto, paridade, idade e instrução da mãe), implantou em 1990, no Brasil, o Sistema de Informações sobre Nascidos Vivos (SINASC), tendo como documento-base a Declaração de Nascido Vivo (DNV) com a finalidade de suprir essas lacunas 7 .

A escassez de dados oficiais no Brasil referentes aos defeitos congênitos aponta para a necessidade do aperfeiçoamento dos sistemas de informação já existentes. Em 1999, tornouse possível o relato dessas informações nas DNV por meio da introdução do campo de número 34 "Detectada alguma malformação congênita el ou anomalia cromossômica?", específico para o registro de tais condições ${ }^{8,9}$. Diante de resposta positiva, o defeito deve ser descrito e codificado segundo a 10ạ revisão da Classificação Internacional de Doenças (CID-10). Tal sistemática possibilita registro bastante completo das alterações 10 .

Tal informação recolhida no total de nascimentos inexiste na maioria dos países. O Brasil está, portanto, na situação privilegiada de contar com um sistema em âmbito nacional para a monitoração e vigilância epidemiológica dos defeitos congênitos 11,12.

Entretanto, o SINASC ainda apresenta limitações como fonte para a determinação da prevalência das anomalias congênitas. A subnotificação ainda é regra e a qualidade na informação registrada precisa melhorar 8,9,13.

Atualmente, um sistema de monitoração dos defeitos congênitos no Brasil, mediante um projeto de pesquisa, é realizado pela rede de maternidades que trabalham em colaboração com o Estudo Colaborativo Latino-Americano de Malformações Congênitas (ECLAMC) e tem sido desenvolvido por diversos profissionais como geneticistas médicos, pediatras e obstetras. As maternidades brasileiras que fazem parte da rede ECLAMC, por outro lado, não cobrem $2 \%$ dos nascimentos do país 14,15,16. Outro programa de vigilância epidemiológica no Brasil é realiza- do pelo Hospital de Clínicas de Porto Alegre da Universidade Federal do Rio Grande do Sul, pelo Programa de Monitorização de Defeitos Congênitos, vinculado ao ECLAMC. O programa mantém um registro ininterrupto dos nascimentos desse hospital (60 mil recém-nascidos avaliados desde 1983), disponibilizando um valioso banco de dados para o desenvolvimento de estudos sobre defeitos congênitos 14,16.

O médico geneticista tem interesse natural pelos defeitos congênitos já que eles representam as ocorrências mais freqüentes em sua atividade profissional. Tal fato faz que seja comum a atuação desses profissionais em serviços de neonatologia, integrando equipes multidisciplinares 17,18. O Município de São Paulo tem 10.886.518 de habitantes (Instituto Brasileiro de Geografia e Estatística. Contagem populacional 2007. http:// www.ibge.gov.br/home/estatistica/populacao/ contagem2007/default.shtm, acessado em 08/ Fev/2009) e conta com 95 maternidades, sendo 53 privadas e 42 públicas. Ocorrem cerca de 190 mil nascimentos por ano (Departamento de Informática do SUS. http://tabnet.datasus.gov.br/ tabnet/tabnet.htm\#EstatVitais, acessado em 08/ Fev/2009). Poucas maternidades contam com um serviço especializado de genética clínica. Entre outras razões, isso ocorre porque no Sistema Único de Saúde (SUS) não há uma política nacional de atenção à saúde em genética clínica, levando as maternidades públicas a ficarem desassistidas 17,18. Uma das conseqüências é que o médico pediatra tem a responsabilidade de reconhecer e diagnosticar todos os defeitos congênitos, mesmo os que representam síndromes genéticas.

Por meio de uma parceria entre a Coordenação de Epidemiologia e Informação da Secretaria Municipal de Saúde de São Paulo e o Centro de Genética Médica da Universidade Federal de São Paulo, foi criado o projeto Qualificação do Preenchimento do Campo 34 da Declaração de Nascido Vivo - Malformação Congênita elou Anomalia Cromossômica para orientar o correto preenchimento do campo 34 da DNV 19.

\section{Objetivo}

Hoje, o sub-registro de defeitos congênitos na DNV é uma realidade que atrapalha o estudo epidemiológico dessas anomalias e prejudica ações em prevenções. Provavelmente, o sub-registro se deve ao desconhecimento de sua importância na saúde pública e o impacto que os defeitos congênitos têm na sociedade como um todo. Portanto, se auxiliarmos os profissionais a diagnosticarem e a notificarem de forma correta os defeitos 
congênitos, poderemos observar esse reflexo no campo 34 da DNV.

O objetivo é acompanhar a evolução do registro dos defeitos congênitos em quatro maternidades do SUS pelo campo 34 da DNV.

\section{Métodos}

Com o apoio dos técnicos e com as informações da Coordenação de Epidemiologia e Informação da Secretaria Municipal de Saúde de São Paulo, utilizamos o banco de dados do SINASC (Departamento de Informática do SUS. http:// www.datasus.gov.br) para acompanhar a evolução do preenchimento do sim, não, sem informação e ignorado do campo 34 da DNV nas quatro maternidades atuantes no estudo e nas demais maternidades do Município de São Paulo. Foram organizados e ministrados cursos de capacitação aos profissionais de saúde de todas as maternidades com dois enfoques: descrição dos defeitos congênitos e codificação baseada na CID-10.

Foi estabelecida uma rede com quatro maternidades inseridas no SUS do Município de São Paulo (Hospital do Servidor Público Municipal, Conjunto Hospitalar do Mandaqui, Hospital Estadual Vila Alpina, Hospital Municipal Maternidade-Escola de Vila Nova Cachoeirinha). Os critérios de inclusão foram: todas as maternidades deveriam ser do SUS, nenhuma das maternidades participantes ter em seu corpo clínico um médico especialista em genética clínica e as maternidades deveriam ter acesso à Internet.

Foi criada uma página on-line (http://www. campo34.med.br) onde o médico pediatra acessava um prontuário eletrônico contendo anamnese (data do nascimento, nome da mãe, nome do pai, nome do recém-nascido, idade da mãe, idade do pai, sexo do recém-nascido, histórico obstétrico, histórico familiar, histórico do parto), exame físico (peso ao nascimento, comprimento ao nascimento, perímetro cefálico ao nascimento, descrição fenotípica do recém-nascido, resultado de exames complementares) e documentação fotográfica (foto da face frente/perfil, do corpo e do defeito congênito em específico). Dados importantes para se fazer um diagnóstico de defeitos congênitos.

Os pediatras das quatro maternidades enviavam os prontuários dos recém-nascidos que apresentavam algum tipo de defeito congênito para o Centro de Genética Médica da Universidade Federal de São Paulo, e este $\boldsymbol{e}$-mail era prontamente respondido com a hipótese diagnóstica do caso. As maternidades deveriam enviar um caso de recém-nascido normal por semana no ano de 2008 como controle de funcionamento do sistema.

Esses $e$-mails foram criptografados com chave de 128 bits. Além disso, o servidor no qual o site foi hospedado contava com proteção firewall e redundâncias. Cada médico pediatra participante do trabalho recebeu uma senha na qual acessava o prontuário. Todas as informações foram criptografadas e armazenadas em um banco de dados eletrônico (desktop específico com trava). Foram realizados backups criptografados de todos os casos recebidos em DVD.

Em todos os casos enviados houve o preenchimento do Termo de Consentimento Livre e Esclarecido no qual os pais dos recém-nascidos declararam ciência. O projeto foi aprovado pelo Comitê de Ética em Pesquisa da Universidade Federal de São Paulo.

\section{Resultados}

Utilizando o prontuário eletrônico on-line, no período de 1 o de janeiro de 2008 a 1o de janeiro de 2009, foram recebidos ao todo 334 casos com suspeitas de defeitos congênitos e 108 controles.

Observamos na Tabela 1, segundo o SINASC, a evolução dos defeitos congênitos registrados no campo 34 da DNV no Município de São Paulo ao longo do tempo.

Observamos na Tabela 2, segundo o SINASC, a evolução dos defeitos congênitos registrados no campo 34 da DNV nas quatro maternidades que utilizaram o sistema on-line.

Observamos na Tabela 3, segundo o SINASC, a evolução dos defeitos congênitos registrados no campo 34 da DNV no Município de São Paulo e em cada maternidade que utilizou o sistema on-line.

\section{Discussão}

O SINASC se propõe a coletar e a produzir informações sobre a ocorrência dos nascimentos no Brasil. O correto preenchimento da DNV é fundamental já que este instrumento é a fonte do sistema de informação 20,21,22.

A DNV pode ser considerada um método de monitoramento de defeitos congênitos no Brasil por causa de algumas características desse documento: é padronizada pelo Ministério da Saúde; de preenchimento obrigatório para todos os recém-nascidos; é de fácil manuseio e qualquer profissional da área de saúde pode preencher os seus 41 campos; utiliza um código padronizado para a classificação das anomalias e tem a supervisão técnica dos cartórios de registro civil e das 
Tabela 1

Registro dos defeitos congênitos no campo 34 da Declaração de Nascido Vivo no Município de São Paulo, Brasil, $2001-2008$.

\begin{tabular}{|c|c|c|c|c|c|c|c|c|c|c|c|c|c|c|c|c|}
\hline & \multicolumn{2}{|c|}{2001} & \multicolumn{2}{|c|}{2002} & \multicolumn{2}{|c|}{2003} & \multicolumn{2}{|c|}{2004} & \multicolumn{2}{|c|}{2005} & \multicolumn{2}{|c|}{2006} & \multicolumn{2}{|c|}{2007} & \multicolumn{2}{|c|}{2008} \\
\hline & $\mathrm{n}$ & $\%$ & $n$ & $\%$ & $\mathrm{n}$ & $\%$ & $\mathrm{n}$ & $\%$ & $\mathbf{n}$ & $\%$ & $\mathrm{n}$ & $\%$ & $\mathrm{n}$ & $\%$ & $\mathbf{n}$ & $\%$ \\
\hline Sim & 830 & 0,42 & 977 & 0,50 & 1.099 & 0,56 & 1.252 & 0,63 & 1.338 & 0,69 & 1.397 & 0,73 & 1.603 & 0,85 & 1.734 & 0,91 \\
\hline Não & 150.039 & 76,16 & 161.145 & 81,90 & 166.835 & 85,24 & 170.790 & 86,58 & 170.242 & 87,67 & 179.870 & 94,32 & 183.977 & 97,42 & 186.437 & 98,33 \\
\hline Ignorado & 21.494 & 10,91 & 13.494 & 6,86 & 4.971 & 2,54 & 4.805 & 2,44 & 6.063 & 3,12 & 1.855 & 0,97 & 987 & 0,52 & 525 & 0,28 \\
\hline $\begin{array}{l}\text { Sem } \\
\text { informação }\end{array}$ & 24.638 & 12,51 & 21.142 & 10,75 & 22.822 & 11,66 & 20.422 & 10,35 & 16.541 & 8,52 & 7.581 & 3,98 & 2.283 & 1,21 & 904 & 0,48 \\
\hline Total & 197.001 & 100,00 & 196.758 & 100,00 & 195.727 & 100,00 & 197.269 & 100,00 & 194.184 & 100,00 & 190.703 & 100,00 & 188.850 & 100,00 & 189.600 & 100,00 \\
\hline
\end{tabular}

Tabela 2

Registro dos defeitos congênitos no campo 34 da Declaração de Nascido Vivo nas quatro maternidades do estudo, $2001-2008$.

\begin{tabular}{|c|c|c|c|c|c|c|c|c|c|c|c|c|c|c|c|c|}
\hline & \multicolumn{2}{|c|}{2001} & \multicolumn{2}{|c|}{2002} & \multicolumn{2}{|c|}{2003} & \multicolumn{2}{|c|}{2004} & \multicolumn{2}{|c|}{2005} & \multicolumn{2}{|c|}{2006} & \multicolumn{2}{|c|}{2007} & \multicolumn{2}{|c|}{2008} \\
\hline & $n$ & $\%$ & $\mathrm{n}$ & $\%$ & $n$ & $\%$ & $\mathrm{n}$ & $\%$ & $\mathrm{n}$ & $\%$ & $\mathrm{n}$ & $\%$ & $\mathrm{n}$ & $\%$ & $n$ & $\%$ \\
\hline Sim & 15 & 0,22 & 69 & 0,76 & 105 & 0,92 & 108 & 0,92 & 125 & 1,12 & 144 & 1,27 & 159 & 1,34 & 249 & 2,02 \\
\hline Não & 5.883 & 84,75 & 7.928 & 87,40 & 9.770 & 85,51 & 10.481 & 88,81 & 10.543 & 94,35 & 11.154 & 98,61 & 11.727 & 98,5 & 12.073 & 97,88 \\
\hline Ignorado & 769 & 11,08 & 838 & 9,24 & 1.534 & 13,43 & 1.210 & 10,25 & 505 & 4,52 & 7 & 0,06 & 11 & 0,09 & 7 & 0,06 \\
\hline $\begin{array}{l}\text { Sem } \\
\text { informação }\end{array}$ & 275 & 3,96 & 236 & 2,60 & 16 & 0,14 & 2 & 0,02 & 1 & 0,01 & 6 & 0,05 & 9 & 0,08 & 4 & 0,03 \\
\hline Total & 6.942 & 100,00 & 9.071 & 100,00 & 11.425 & 100,00 & 11.801 & 100,00 & 11.174 & 100,00 & 11.311 & 100,00 & 11.906 & 100,00 & 12.334 & 100,00 \\
\hline
\end{tabular}

Tabela 3

Registro dos defeitos congênitos no campo 34 da Declaração de Nascido Vivo no Município de São Paulo, Brasil, e nas quatro maternidades do estudo, 20012008.

\begin{tabular}{|c|c|c|c|c|c|c|c|c|c|c|c|c|c|c|c|c|}
\hline & \multicolumn{2}{|c|}{2001} & \multicolumn{2}{|c|}{2002} & \multicolumn{2}{|c|}{2003} & \multicolumn{2}{|c|}{2004} & \multicolumn{2}{|c|}{2005} & \multicolumn{2}{|c|}{2006} & \multicolumn{2}{|c|}{2007} & \multicolumn{2}{|c|}{2008} \\
\hline & $\mathrm{n}$ & $\%$ & $n$ & $\%$ & $\mathrm{n}$ & $\%$ & $n$ & $\%$ & $\mathrm{n}$ & $\%$ & $\mathrm{n}$ & $\%$ & $\mathrm{n}$ & $\%$ & $\mathrm{n}$ & $\%$ \\
\hline São Paulo & 830 & 0,42 & 977 & 0,50 & 1.099 & 0,56 & 1.252 & 0,63 & 1.338 & 0,69 & 1.397 & 0,73 & 1.603 & 0,85 & 1.734 & 0,91 \\
\hline Hospital do & 3 & 0,23 & 3 & 0,24 & 4 & 0,31 & 3 & 0,23 & 6 & 0,51 & 15 & 1,39 & 9 & 0,93 & 31 & 3,46 \\
\hline \multicolumn{17}{|l|}{ Servidor } \\
\hline \multicolumn{17}{|l|}{ Público } \\
\hline \multicolumn{17}{|l|}{ Municipal } \\
\hline Conjunto & 7 & 0,23 & 17 & 0,62 & 19 & 0,64 & 13 & 0,46 & 32 & 1,20 & 26 & 0,92 & 35 & 1,14 & 55 & 1,62 \\
\hline \multicolumn{17}{|l|}{ Hospitalar do } \\
\hline \multicolumn{17}{|l|}{ Mandaqui } \\
\hline Hospital & 0 & 0,00 & 5 & 0,24 & 4 & 0,15 & 9 & 0,37 & 10 & 0,45 & 3 & 0,13 & 5 & 0,20 & 31 & 1,49 \\
\hline \multicolumn{17}{|l|}{ Estadual Vila } \\
\hline \multicolumn{17}{|l|}{ Alpina } \\
\hline Hospital & 5 & 0,19 & 44 & 1,46 & 78 & 1,70 & 83 & 1,58 & 77 & 1,50 & 100 & 1,93 & 110 & 2,06 & 132 & 2,21 \\
\hline \multicolumn{17}{|l|}{ Municipal } \\
\hline \multicolumn{17}{|l|}{ Maternidade- } \\
\hline \multicolumn{17}{|l|}{ Escola de } \\
\hline \multicolumn{17}{|l|}{ Vila Nova } \\
\hline Cachoeirinha & & & & & & & & & & & & & & & & \\
\hline
\end{tabular}


secretárias de saúde municipais por fornecer e controlar a numeração dos impressos.

A DNV tem uma importância crucial para o acompanhamento de políticas de saúde pública na área da genética médica, obstetrícia, pediatria e na área preventiva de defeitos congênitos, como por exemplo, a fortificação do ácido fólico nas farinhas para prevenir os defeitos de fechamento do tubo neural e o rastreamento de anomalias provocadas por uso de medicações teratogênicas como a talidomida ou o misoprostol 14,23,24,25. Um sistema deste tipo poderá esclarecer prontamente e a baixo custo suspeitas de epidemias de defeitos congênitos como a presenciada na década de 1980 na cidade de Cubatão, São Paulo, cuja elucidação demandou esforço continuado de uma série de profissionais durante cinco anos 26,27,28.

Podemos utilizar outros dados relevantes da DNV como o número de consultas de pré-natal, a idade materna, a duração gestacional, o tipo de gravidez, o tipo do parto, o peso ao nascer, o sexo, o índice Apgar e a raça/cor 29,30,31.

O sub-registro dos defeitos congênitos no campo 34 da DNV é uma verdade válida para praticamente todos os municípios do Brasil, excetuando alguns municípios onde existem programas de monitoramento e pesquisa. Isso gera um problema quando queremos analisar epidemiologicamente a incidência e os tipos de defeitos congênitos na população de nascidos vivos. O Município de São Paulo, o mais populoso do país, com um número elevado de nascimentos ao ano, com um número elevado de maternidades (públicas e privadas) e cada uma dessas com suas heterogeneidades de trabalho, não foge à regra $\mathrm{e}$ apresenta um sub-registro de defeitos congênitos ao longo dos anos desde que foi incorporado o campo 34 na DNV.

Acredita-se que a maioria dos defeitos congênitos é reconhecida na criança nas primeiras horas de vida pelos profissionais da saúde, sendo o principal problema o registro da informação no próprio campo 34 da DNV e sua classificação de acordo com o CID-10.

Com o projeto que o Centro de Genética Médica da Universidade Federal de São Paulo desenvolve com a Secretaria Municipal de Saúde de São Paulo, iniciado no ano de 2005, a totalidade das maternidades da cidade de São Paulo foram abordadas, mais de uma centena de profissionais na área de saúde entre médicos, enfermeiros e escriturários foram orientados sobre o que é um defeito congênito e como preencher de forma correta o campo 34 da DNV.

Notamos que a notificação dos defeitos congênitos no Município de São Paulo vem melhorando ano a ano. O registro do campo sim, que em 2004 era de 0,63\%, vem aumentando de maneira gradativa passando a 0,69\% em 2005, 0,73\%, em 2006, 0,85\%, em 2007 e finalmente com 0,91\% no ano de 2008. Mas o registro ainda apresentase abaixo do que se espera, ou seja, uma taxa de $2 \%$ a $3 \%$. Outro item importante no registro dos defeitos congênitos é o número alto de DNV com os campos sem informação e ignorado. Em 2004, o número de DNV sem informação e ignoradas juntas eram de 12,79\% do total e já em 2008 era de $0,76 \%$. Percebe-se assim que o sistema está sendo aperfeiçoado com o passar do tempo.

Uma das ações centrais deste estudo levadas a efeito para contribuir com esta tendência foi a capacitação dos médicos pediatras, dos enfermeiros e da administração para realizarem o diagnóstico da maioria das anomalias congênitas e mostrar a importância do registro dos defeitos congênitos.

A partir de 2005, observamos melhora no registro de defeitos congênitos no campo 34 nas quatro maternidades. O número de registros de sim vem aumentando de uma maneira geral e há redução do número de notificação dos campos sem informação e ignorados. Mas a partir do ano de 2008, quando se inicia o uso do prontuário eletrônico, notamos uma melhora significativa dos registros de sim. No Hospital do Servidor Público Municipal, o registro de defeitos congênitos em 2004 era de $0,23 \%$, em 2007 registravase $0,93 \%$ e em 2008 aumentou para $3,46 \%$. No Conjunto Hospitalar do Mandaqui, em 2004 era de $0,46 \%$, em 2007 de 1,14\% e em 2008 foi para 1,62\%. No Hospital Estadual Vila Alpina, em 2004 era de 0,37\%, em 2007 registrou 0,2\% e em 2008 passou para $1,49 \%$. E na Maternidade de Vila Nova Cachoeirinha, em 2004, o registro de defeitos congênitos estava no patamar ideal de 1,58\%, em 2007 subiu para 2,06\% e em 2008 houve uma elevação de $0,15 \%$ indo para 2,21\%. Percebemos que em 2008 as quatro maternidades registraram freqüências acima de $1 \%$, porém há heterogeneidade entre elas ( $p<0,01)$. Assim, mesmo com métodos uniformes aplicados nas maternidades o desempenho foi variável.

Se analisarmos todas as quatro maternidades como se fossem um único estabelecimento, antes do projeto em 2004, a taxa de registro de defeitos congênitos era de 0,92\%. Em 2007, tal taxa elevase para $1,34 \%$ e depois do uso do prontuário eletrônico a taxa sobe para 2,02\% (p<0,001).

Verificamos também a redução do número de sem informação e ignorados quando comparados com o ano anterior.

O sistema de transmissão de dados pela Internet foi o sistema escolhido para auxiliar o médico pediatra nas maternidades e se mostrou um sistema prático, rápido e seguro. Durante o período 
do trabalho não houve nenhum tipo de interferência externa (bugs) ou mau funcionamento do sistema. Para manter um fluxo de informação constante, instituímos a rotina pela qual cada maternidade enviava todos os dados do prontuário de uma criança sem defeitos congênitos, os quais chamamos controle, semanalmente. $\mathrm{O}$ sistema de referência e contra-referência funcionou a contento.

A praticidade se deve ao fato de que tentamos aproximar ao máximo o prontuário eletrônico a uma ficha de anamnese comum, com dados objetivos e de fácil preenchimento. Simulamos o envio de fotos como se anexássemos documentos a um e-mail comum.

A agilidade se deve ao fato de que no mesmo dia o caso era enviado ao centro de referência e se sabia, então, qual o tipo de anomalia, qual sua gravidade, em qual maternidade o recémnascido nasceu e qual o médico responsável pelo caso.

\section{Conclusão}

Com uma intervenção educativa nos berçários do Município de São Paulo houve melhora no registro dos defeitos congênitos na DNV ao longo dos anos. Entretanto, esse registro $(1,07 \%)$ ainda esta abaixo do esperado ( $2 \%$ a $3 \%$ ).

A DNV pode se tornar um excelente sistema de monitoração e vigilância para os defeitos congênitos, não só no Município de São Paulo, mas no Brasil de uma maneira geral.

A orientação e a capacitação de profissionais na área de saúde sobre a importância e o funcionamento da DNV é um modelo eficaz para a melhora do registro dos defeitos congênitos.

O prontuário eletrônico que utiliza a Internet como meio de comunicação, mostrou-se eficaz no auxílio aos pediatras para diagnosticar os defeitos congênitos.

$\mathrm{O}$ projeto mostrou que, se as maternidades estabelecerem parcerias com centros especializados no diagnóstico e registros de defeitos congênitos na DNV, poderemos abreviar o tempo para termos registros confiáveis de anomalias congênitas no Brasil. Tal parceria com serviços de genética poderá também melhorar a assistência em termos de reconhecimento precoce de síndromes genéticas e a conseqüente intervenção em termos preventivos por intermédio de aconselhamento genético para as famílias e assistenciais para o paciente.

\section{Resumo}

O objetivo foi aumentar a freqüência da notificação de anomalias congênitas no campo 34 da Declaração de Nascido Vivo em quatro maternidades do Município de São Paulo, Brasil, ao longo do ano de 2008. Utilizamos o banco de dados do Sistema de Informações sobre Nascidos Vivos da Secretária Municipal de Saúde de São Paulo para acompanhar a evolução dos registros dos defeitos congênitos. Mediante prontuário eletrônico, via Internet, os casos suspeitos eram enviados para um centro de referência em genética médica. O prontuário eletrônico contém anamnese, exame físico e fotos do recém-nascido. O estudo ocorreu em quatro maternidades com uma amostra total de 10 mil nas cimentos no ano e que não apresentam médico geneticista. Houve aumento da notificação dos defeitos congênitos nas quatro maternidades onde o estudo foi realizado quando comparado com os anos anteriores e com o registro do Município de São Paulo. O método de referência e contra-referência utilizando a Internet mostrou-se eficaz.

Anormalidades Congênitas; Notificação de Doenças; Declaração de Nascimento; Internet; Sistemas de Informação 


\section{Colaboradores}

P. D. R. De Nicola participou na coleta, análise e interpretação dos dados, além da redação do artigo. M. C. S. P. Cernach colaborou na revisão crítica do artigo. A. B. A. Perez contribuiu na análise e interpretação de dados. D. Brunoni idealizador do trabalho, participou da análise e interpretação de dados.

\section{Agradecimentos}

À Coordenação de Epidemiologia e Informação (CEInfo) da Secretária Municipal de Saúde de São Paulo, ao Centro de Genética Médica da Universidade Federal de São Paulo, ao Hospital do Servidor Público Municipal, ao Conjunto Hospitalar do Mandaqui, ao Hospital Estadual Vila Alpina e ao Hospital Municipal MaternidadeEscola de Vila Nova Cachoeirinha "Dr. Mário de Moraes Altenfelder Silva".

\section{Referências}

1. Organização Pan-Americana da Saúde. Prevenção e controle de enfermidades genéticas e os defeitos congênitos: relatório de um grupo de consulta. Washington DC: Organização Pan-Americana da Saúde; 1984

2. Leite JCL, Schuler-Faccini L. Defeitos congênitos em uma região de mineração de carvão. Rev Saúde Pública 2001; 35:136-41.

3. McKusick V. The growth and development of human genetics as a clinical discipline. Am J Hum Genet 1975; 27:261-73.

4. Laurenti R. A mortalidade por malformação congênita no município de São Paulo no período de 1940 a 1964. Rev Saúde Pública 1967; 1:51-8.

5. Szwarcwald CL, Leal MC, Castilho EA, Andrade CLT. Mortalidade infantil no Brasil: Belíndia ou Bulgária? Cad Saúde Pública 1997; 13:503-16.

6. Geremias AL, Almeida MF, Flores LPO. Avaliação das declarações de nascido vivo como fonte de informação sobre defeitos congênitos. Rev Bras Epidemiol 2009; 12:60-8.

7. Mello Jorge MHP, Gotlieb SLD, Soboll MLMS, Almeida MF, Latorre MRDO. Avaliação do sistema de informação sobre nascidos vivos e o uso de seus dados em epidemiologia e estatísticas de saúde. Rev Saúde Pública 1993; 27 Suppl:1-46.

8. Guerra FAR, Llerena Jr. JC, Gama SGN, Cunha CB, Theme Filha MM. Defeitos congênitos no Município do Rio de Janeiro, Brasil: uma avaliação através do SINASC (2000-2004). Cad Saúde Pública 2008; 24:140-9.

9. Guerra FAR, Llerena Jr. JC, Gama SGN, Cunha CB, Theme Filha MM. Confiabilidade das informações das declarações de nascido vivo com registro de defeitos congênitos no Município do Rio de Janeiro, Brasil, 2004. Cad Saúde Pública 2008; 24:438-46.

10. Horovitz DDG, Llerena Jr. JC, Mattos RA. Atenção aos defeitos congênitos no Brasil: panorama atual. Cad Saúde Pública 2005; 21:1055-64.

11. Ribeiro AM, Guimarães MJ, Lima MC, Sarinho SW, Coutinho SB. Fatores de risco para mortalidade neonatal em crianças com baixo peso ao nascer. Rev Saúde Pública 2009; 43:246-55.

12. Almeida MF, Novaes HMD, Alencar GP, Rodrigues LC. Mortalidade neonatal no Município de São Paulo: influência do peso ao nascer e de fatores sócio-demográficos e assistenciais. Rev Bras Epidemiol 2002; 5:93-107.

13. Luquetti DV, Koifman RJ. Quality of reporting on birth defects in birth certificates: case study from a Brazilian reference hospital. Cad Saúde Pública 2009; 25:1721-31.

14. Horovitz DDG, Cardoso MHCA, Llerena Jr. JC, Mattos RA. Atenção aos defeitos congênitos no Brasil: características do atendimento e propostas para formulação de políticas públicas em genética clínica. Cad Saúde Pública 2006; 22:2599-609.

15. Castilla EE, Orioli IM. ECLAMC: the Latin-American collaborative study of congenital malformations. Community Genet 2004; 7:76-94.

16. Schuler-Faccini L, Leite JCL, Sanseverino MTV, Peres RM. Avaliação de teratógenos potenciais na população brasileira. Ciênc Saúde Coletiva 2002; 7:65-71. 
17. Brunoni D. Aconselhamento genético. Ciênc Saúde Coletiva 2002; 7:101-7.

18. Brunoni D. Estado atual do desenvolvimento dos serviços de genética médica no Brasil. Rev Bras Genét 1997; 20 Suppl:11-23.

19. Coordenação de Epidemiologia e Informação, Secretaria Municipal de São Paulo. Declaração de Nascido Vivo: campo 34 - manual de anomalias congênitas. São Paulo: Secretaria Municipal de São Paulo; 2008.

20. Fundação Nacional de Saúde. Manual de instruções para o preenchimento da declaração de nascido vivo. 3a Ed. Brasília: Fundação Nacional de Saúde; 2001.

21. Coordenação de Epidemiologia e Informação, Secretaria Municipal de São Paulo. Manual de orientações para codificação e digitação de endereço de residência no Sistema de Informação sobre Nascidos Vivos - SINASC. São Paulo: Secretaria Municipal de São Paulo; 2007.

22. Coordenação de Epidemiologia e Informação, Secretaria Municipal de São Paulo. Declaração de Nascido Vivo: manual de preenchimento. São Paulo: Secretaria Municipal de São Paulo; 2008.

23. Pacheco SS, Braga C, Souza AI, Figueiroa JN. Efeito da fortificação alimentar com ácido fólico na prevalência de defeitos do tubo neural. Rev Saúde Pública 2009; 43:565-71.

24. Osorio-de-Castro CGS, Paumgartten FJR, Silver LD. O uso de medicamentos na gravidez. Ciênc Saúde Coletiva 2004; 9:987-96.
25. Oliveira MA, Bermudez JAZ, Souza ACM. Talidomida no Brasil: vigilância com responsabilidade compartilhada? Cad Saúde Pública 1999; 15:99-112.

26. Monteleone-Neto R, Brunoni D, Laurenti R. Projeto Cubatão: uma proposta em aberto para a comunidade científica. Ciênc Hoje 1982; 1:14-5.

27. Monteleone-Neto R, Brunoni D, Laurenti R, Mello Jorge MHP, Gotlieb SL, Lebrão ML. Birth defects and environmental pollution: the Cubatão example. Prog Clin Biol Res 1985; 163B:65-8.

28. Monteleone-Neto R, Castilla EE. Apparently normal frequency of congenital anomalies in the highly polluted town of Cubatão, Brazil. Am J Med Genet 1994; 52:319-23.

29. Romero DE, Cunha CB. Avaliação da qualidade das variáveis epidemiológicas e demográficas do Sistema de Informações sobre Nascidos Vivos, 2002. Cad Saúde Pública 2007; 23:701-14.

30. Silva AAM, Ribeiro VS, Borba Júnior AF, Coimbra LC, Silva RA. Avaliação da qualidade dos dados do Sistema de Informações sobre Nascidos Vivos em 1997-1998. Rev Saúde Pública 2001; 35:508-14.

31. Theme Filha MM, Gama SGN, Cunha CB, Leal MC. Confiabilidade do Sistema de Informações sobre Nascidos Vivos Hospitalares no Município do Rio de Janeiro, 1999-2001. Cad Saúde Pública 2004; 20 Suppl 1:S83-91.

Recebido em 23/Nov/2009

Versão final reapresentada em 31/Mar/2010 Aprovado em 05/Abr/2010 